\title{
Change in Maximal Esophageal Wall Thickness Provides Prediction of Survival and Recurrence in Patients with Esophageal Squamous Cell Carcinoma After Neoadjuvant Chemoradiotherapy and Surgery
}

This article was published in the following Dove Press journal:

Cancer Management and Research

Yahua Wu (D)

Jiancheng $\mathrm{Li}$

Department of Radiation Oncology, Fujian Medical University Cancer Hospital, Fujian Cancer Hospital, Fuzhou, Fujian, 3500 I4, People's Republic of China
Correspondence: Jiancheng Li Department of Radiation Oncology, Fujian Medical University Cancer

Hospital, Fujian Cancer Hospital, Jin'an District, Fuzhou, Fujian, 3500I4, People's Republic of China

Tel +8613906900190

Email jianchengli_jack@I26.com
Purpose: This study aimed to evaluate the relationship of the percentage decrease of maximal esophageal wall thickness with pathological complete response (pCR) and recurrence in esophageal squamous cell carcinoma (ESCC).

Patients and Methods: A total of 146 ESCC patients treated with neoadjuvant chemoradiotherapy (NCRT) and surgery were included. The prognostic factors for overall survival (OS) and disease-free survival (DFS) were analyzed. The recurrence site, time, and frequency were included in the analysis. The percentage decrease of maximal esophageal wall thickness after NCRT was determined with the formula: [(pre-post)/pre] $\times 100$.

Results: Overall, only 42 patients achieved pCR. Multivariable logistic analyses showed that the percentage decrease of maximal esophageal wall thickness (HR: 2.504; 95\% CI: 1.112-5.638, $\mathrm{P}=0.027$ ) was independently correlated with $\mathrm{pCR}$. In multivariable Cox analyses, a $\leq 40 \%$ percentage decrease of maximal esophageal wall thickness was an independent adverse factor for both OS (HR: 1.907, 95\% CI: 1.149-3.165; P=0.012) and DFS (HR: 2.054, 95\% CI: 1.288-3.277; $\mathrm{P}=0.003$ ). Compared with patients with a $\leq 40 \%$ percentage decrease, those with a $>40 \%$ percentage decrease had better 5 -year OS $(29.0 \%$ vs $60.1 \%$, $\mathrm{P}<0.05)$ and DFS $(27.8 \%$ vs $54.4 \%, \mathrm{P}<0.05)$. Perineural invasion (PNI) was also an unfavorable factor for OS (HR: 2.138, 95\% CI: $0.094-4.178$; $\mathrm{P}=0.026$ ). Lymph vessel invasion (HR: 2.874, 95\% CI: $1.574-5.248 ; \mathrm{P}=0.001$ ) and $\mathrm{PNI}(\mathrm{HR}$ : 2.050; 95\% CI: 1.044 4.023; $\mathrm{P}=0.037$ ) were independent prognosticators for DFS. The rates of local and distant recurrence were also significantly difference between those with a percentage decrease of $\leq 40 \%$ and of $>40 \%(\mathrm{P}<0.05)$.

Conclusion: The percentage decrease of maximal esophageal wall thickness is associated with pCR and recurrence in ESCC patients who undergo NCRT and surgery and can thus be used to independently predict prognosis.

Keywords: neoadjuvant, esophageal squamous cell carcinoma, esophageal wall thickness, pathological complete response, recurrence

\section{Introduction}

Esophageal cancer (EC) remains to be a common malignancy and a leading cause of cancer death worldwide, with approximately more than 572,000 incident cases and 508,000 related deaths reported in 2018. ${ }^{1}$ Esophageal squamous cell carcinoma (ESCC) is the predominant histological subtype, accounting for more than $85 \%$ of cases. $^{2}$ The mortality and incidence rates of ESCC are currently higher in East Asian countries, especially in China. ${ }^{3}$ Previously, surgery alone was the standard 
primary treatment modality for early ESCC, offering a 5-year survival rate of up to $80 \%$. However, surgery alone yields poor outcomes, and the mortality rate is up to $5 \%$ for locally advanced ESCC. ${ }^{4-6}$ At present, surgery combined with neoadjuvant chemotherapy (NCRT) has become the standard treatment for locally advanced ESCC, based on the results of the CROSS trial. Approximately $20-51 \%$ of ESCC patients treated with NCRT achieve pathological complete response (pCR), and these patients survive longer than those with a nonpCR. $^{7-9}$ Considering the complexity and risks of esophagectomy, surgery may be unnecessary for patients who achieve pCR after NCRT, and active surveillance may be a safer approach in these patients. ${ }^{10}$ However, effective factors to accurately predict pCR are lacking.

Computed tomography (CT) is frequently used for patient assessment before and after chemoradiotherapy because it is easy to perform. Accordingly, we envisioned whether meaningful information to predict $\mathrm{pCR}$ and recurrences can be obtained from CT. Previous studies have evaluated treatment benefits according to changes in the maximum esophageal wall thickness on CT. Swisher et al showed that the maximal esophageal wall thickness after chemoradiotherapy was corrected with treatment response. $^{11}$ Further, $\mathrm{Li}$ et al found that a prechemoradiotherapy maximal esophageal wall thickness of $\geq 20 \mathrm{~mm}$ predicts $\mathrm{pCR}$ and better survival after chemoradiotherapy. ${ }^{12}$ However, there are few studies on the predictive value of the percentage decrease of maximal esophageal wall thickness for ESCC treated with NCRT. Hence, we aimed to assess the relationship of the percentage decrease of maximal esophageal wall thickness with the probability of $\mathrm{pCR}$ and recurrence in patients with ESCC.

\section{Patients and Methods Study Design and Patients}

This was a retrospective study of 146 patients with locally advanced ESCC who underwent neoadjuvant chemoradiotherapy followed by surgery at Fujian Cancer Hospital between February 2009 and December 2019. The eligibility criteria were as follows: (1) histologically confirmed locally advanced squamous cell carcinoma of the thoracic esophagus; (2) no history of malignancy or second primary tumor; (3) age 18-70 years; (4) Karnofsky performance status score $\geq 70$; and (7) absence of severe organic disease. In total, 13 patients were excluded because of (1) other primary cancer history or a second malignancy $(\mathrm{n}=6)$; (2) a survival time $<1$ month after surgery $(\mathrm{n}=3)$; and (3) loss to follow-up ( $n=8)$. All patients were staged according to the 8th edition of the American Joint Committee on Cancer TNM staging system.

\section{Treatment}

The median dose of neoadjuvant radiotherapy was 40 (range, 36-50.4) Gy in 1.8-20 Gy per fraction, 5 days per week. Overall, 70 patients underwent intensitymodulated radiotherapy and 8 underwent threedimensional conformal radiotherapy. The patient was placed in the supine position, fixed with a vacuum bag or styrofoam, and underwent CT simulation for positioning. The gross tumor volume, clinical target volume, and planning target volume were all delineated according to the National Comprehensive Cancer Network criteria. There were 68 patients who underwent two-dimensional conventional radiotherapy that used the anterior and posterior opposing techniques.

Neoadjuvant chemotherapy comprised a two-drug combination regimen based on platinum and was administered for at least 1 cycle of full-dose chemotherapy. The chemotherapeutics included (1) paclitaxel $135 \mathrm{mg} / \mathrm{m} 2 \mathrm{D} 1$ or docetaxel $75 \mathrm{mg} / \mathrm{m} 2 \mathrm{D} 1$ + cisplatin or nedaplatin $75 \mathrm{mg} / \mathrm{m} 2$ D2 and (2) 5-fluorouracil (5-FU) $700-1000 \mathrm{mg} / \mathrm{m} 2 \mathrm{D} 1-2$ + cisplatin $75 \mathrm{mg} / \mathrm{m} 2$ D2 were administered every 3 weeks. Surgery was conducted 4 to 6 weeks after the end of neoadjuvant chemoradiotherapy. All surgical procedures included three-field lymph node dissection, except for esophagectomy.

\section{Computed Tomography}

CT was performed using a Philips 256-slice spiral CT scanner (Philips Netherlands) at $120 \mathrm{kV}$ and $350 \mathrm{mAs}$. The first CT scans were performed to evaluate the maximal esophageal wall thickness within 1 month before neoadjuvant chemoradiotherapy, and the second scans were performed approximately 4-6 weeks after the completion of CRT. The scan range included the neck, double locks, chest, and upper abdomen. We injected $100 \mathrm{~mL}$ of a non-ionic iodine contrast agent (iohexol) from the cubital vein at a speed of $3 \mathrm{~mL} / \mathrm{s}$. Arterial and venous imaging was performed at 40 and 70 seconds after the injection, and we used a $0.625 \mathrm{~mm}$ image to compare the original venous phase. The images were reconstructed in the axial, coronal, and sagittal positions and were transmitted to the PACS workstation. Image analysis was independently performed by two experienced radiologists 
who measured the maximum esophageal wall thickness. Discrepancies were resolved by involving a third radiologist.

\section{Follow-Up}

All patients were followed up until death or the last follow-up. Assessments were performed regularly every 3 months in the first year, every 6 months for the next 2 years, and once a year thereafter. Follow-up assessments included physical examination, blood routine, biochemistry, tumor markers, CT, and esophageal barium.

\section{Endpoints}

The primary endpoint was overall survival (OS), and the secondary endpoint was disease-free survival (DFS). OS was calculated as the time from the date of the surgery to the date of any-cause death or the date of the last followup. DFS was defined as the time from after surgery to the first recurrence or cancer-related death. Local recurrence was defined as recurrence in the primary tumor site or locoregional lymph nodes. Recurrence in lymph nodes in the abdominal trunk or supraclavicular area was considered to be regional lymph node recurrence, which was also categorized as local recurrence. Distant recurrence was defined as non-regional lymph node recurrence or systemic metastasis.

\section{Statistical Analysis}

Categorical data were compared using the chi-square test or the Fisher exact test, while continuous data were compared using the Wilcoxon-Mann-Whitney nonparametric test. Survival curves were generated using the KaplanMeier method and compared using the Log rank test. Significant factors in the univariate analysis (ie, $\mathrm{P}<0.10$ ) were included in the multivariate Cox analysis to identify independent predictors of survival. Multivariable logistic analyses was uesd to identify factors associated with pCR. All statistical analyses were performed using SPSS 25.0 statistical software (version 25.0; IBM Inc., Armonk, NY, USA). All tests were two-sided, and $\mathrm{P}<0.05$ was considered statistically significant.

\section{Results}

\section{Patient Characteristics}

Of the 146 patients included, 128 (87.7\%) were male, and 18 $(12.3 \%)$ were female. The median patient age was 57 years (range, 38-71 years). There were 40, 85, and 21 patients with upper, middle, and lower esophageal cancer, respectively. The patient characteristics are listed in Table 1. The median percentage decrease of maximal esophageal wall thickness after NCRT was 40\% (range, 0-82.6\%). Overall, 35 (24.0\%) and 111 (76.0\%) patients underwent $<40$ Gy and $\geq 40$ Gy RT, respectively. Most patients had cT3 (65.8\%) and $\mathrm{cN}+$ (81.5\%) disease. A total of 19 (13.0), 79 (54.1), and 48 (32.9) patients were diagnosed with stage II, III, and IV disease, respectively.

Adjuvant chemotherapy was administered in $64 / 146$ (43.8\%) patients.

\section{Factors Associated with Pathological Complete Response}

A total of 42 patients achieved pCR, while 104 patients achieved non-pCR. The pCR group demonstrated better 5 -year OS (78.6\% vs $44.8 \%, \mathrm{P}<0.005)$ and DFS $(77.0 \%$ vs $35.2 \%, \mathrm{P}<0.005)$ than did the non-pCR group (Figure 1). There were significantly more females in the $\mathrm{pCR}$ group than in the non-pCR $(\mathrm{P}=0.039)$. Further, patients with a higher percentage decrease of maximal esophageal wall thickness and higher radiation dose were more likely to achieve pCR. Additionally, patients who achieved pCR were less likely to have lymph vessel invasion (LVI) and perineural invasion (PNI) in the final esophagectomy than non-pCR patients.

Multivariable logistic analyses (Table 2) showed that the percentage decrease of maximal esophageal wall thickness (HR: 2.226; 95\% CI: $1.005-4.930, \mathrm{P}=0.049$ ) and sex (HR: 0.283 ; 95\% CI: $0.097-0.827, \mathrm{P}=0.021$ ) were independently correlated with achieving pCR. Further, the pCR rate was higher in those who received $\geq 40$ Gy than those who received $<40$ Gy (33.3\% vs $14.3 \%$ ), but the difference was not significant $(\mathrm{P}=0.057)$. Patients with a $>40 \%$ decrease of maximal esophageal wall thickness showed a significantly higher $\mathrm{pCR}$ rate compared with those with $\leq 40 \%$ decrease $(35.4 \%$ vs $20.9 \%, \mathrm{P}=0.049)$.

\section{Factors Associated with Survival}

The median follow-up time was 53 months. The results of the univariate and multivariate Cox regression analyses of the influencing factors of OS are shown in Table 3. Univariate analysis revealed that the percentage decrease of maximal esophageal wall thickness, ypT stage, ypN stage, ypTNM, PNI, and LVI were significant prognostic factors associated with $\mathrm{OS}(\mathrm{P}<0.05$ for all). Factors that were highly correlated were not included in the 
Table I Patient Characteristics

\begin{tabular}{|c|c|c|c|c|}
\hline Characteristics & Total $(n=146), \%$ & $\operatorname{pCR}(n=42), \%$ & Non-pCR $(n=104), \%$ & $\mathbf{P}$ \\
\hline Age & & & & 0.992 \\
\hline$\leq 55$ & $59(40.4)$ & $17(40.5)$ & $42(40.4)$ & \\
\hline$>55$ & $87(59.6)$ & $25(59.5)$ & $62(59.6)$ & \\
\hline Sex & & & & 0.039 \\
\hline Male & I28(87.7) & $33(78.6)$ & $95(91.3)$ & \\
\hline Female & $18(12.3)$ & $9(21.4)$ & $9(8.7)$ & \\
\hline KPS & & & & 0.717 \\
\hline Median (range) & $80(70-90)$ & $80(70-90)$ & $80(70-90)$ & \\
\hline Smoking history & & & & 0.231 \\
\hline Yes & $74(50.7)$ & $18(42.9)$ & $56(53.8)$ & \\
\hline No & $72(49.3)$ & $24(57.1)$ & $48(46.2)$ & \\
\hline Tumor location & & & & 0.189 \\
\hline Upper & $40(27.4)$ & $16(38.1)$ & $24(23.1)$ & \\
\hline Middle & $85(58.2)$ & $2 I(50.0)$ & $64(6 \mid .5)$ & \\
\hline Distal & $21(14.4)$ & $5(11.9)$ & $16(15.4)$ & \\
\hline Decrease of maximal esophageal wall thickness (\%) & & & & 0.055 \\
\hline$\leq 40 \%$ & $67(45.9)$ & $14(33.3)$ & $53(50.1)$ & \\
\hline$>40 \%$ & $79(54.1)$ & $28(66.7)$ & $5 I(49.0)$ & \\
\hline Clinical T stage & & & & 0.217 \\
\hline $\mathrm{T} 2$ & $3(2.1)$ & $\mathrm{I}(2.4)$ & $2(1.9)$ & \\
\hline T3 & $96(65.8)$ & $32(76.2)$ & $64(6 \mid .5)$ & \\
\hline $\mathrm{T} 4$ & $47(32.2)$ & $9(21.4)$ & $38(36.5)$ & \\
\hline Clinical $N$ stage & & & & 0.859 \\
\hline No & $27(18.5)$ & $9(21.4)$ & $18(17.3)$ & \\
\hline $\mathrm{NI}$ & $70(47.9)$ & $2 I(50.0)$ & $49(47.1)$ & \\
\hline N2 & $45(30.8)$ & $\mathrm{II}(26.2)$ & $34(32.7)$ & \\
\hline N3 & $4(2.7)$ & $\mathrm{I}(2.4)$ & $3(2.9)$ & \\
\hline Clinical TNM stage & & & & 0.208 \\
\hline II & $19(13.0)$ & $8(19.0)$ & $\mathrm{II}(10.6)$ & \\
\hline III & $79(54.1)$ & $24(57.1)$ & $55(52.9)$ & \\
\hline IV & $48(32.9)$ & $10(23.8)$ & $38(36.5)$ & \\
\hline
\end{tabular}

(Continued) 
Table I (Continued).

\begin{tabular}{|c|c|c|c|c|}
\hline Characteristics & Total $(n=146), \%$ & PCR $(n=42), \%$ & Non-pCR $(n=104), \%$ & $\mathbf{P}$ \\
\hline Radiation dose (Gy) & & & & 0.030 \\
\hline$<40$ & $35(24.0)$ & $5(11.9)$ & $30(28.8)$ & \\
\hline$\geq 40$ & $1 \mathrm{II}(76.0)$ & $37(88.1)$ & $74(7 \mid .2)$ & \\
\hline Radiotherapy modality & & & & 0.497 \\
\hline 2DCR & $68(46.6)$ & $22(52.4)$ & $46(44.2)$ & \\
\hline $3 D C R$ & $8(5.5)$ & $3(7.1)$ & $5(4.8)$ & \\
\hline IMRT & $70(47.9)$ & $17(40.5)$ & $53(51.0)$ & \\
\hline Chemotherapy cycle & & & & 0.552 \\
\hline I & $47(32.2)$ & $12(28.6)$ & $35(33.7)$ & \\
\hline$\geq 2$ & $99(67.8)$ & $30(71.4)$ & $69(66.3)$ & \\
\hline Number of lymph node examined & & & & 0.895 \\
\hline Median (range) & $25(3-75)$ & $24(6-48)$ & $25(3-75)$ & \\
\hline Lymph vessel invasion & & & & $<0.001$ \\
\hline Yes & $28(19.2)$ & $\mathrm{I}(2.4)$ & $27(26.0)$ & \\
\hline No & $118(80.8)$ & $4 I(97.6)$ & $77(74.0)$ & \\
\hline Perineural invasion & & & & 0.001 \\
\hline Yes & $20(13.7)$ & 0 & $20(19.2)$ & \\
\hline No & $126(86.3)$ & $42(100)$ & $84(80.8)$ & \\
\hline Adjuvant chemotherapy & & & & 0.003 \\
\hline Yes & $64(43.8)$ & $10(23.8)$ & $54(51.9)$ & \\
\hline No & $82(56.2)$ & $32(76.2)$ & $50(48.1)$ & \\
\hline
\end{tabular}

Notes: While $N \geq 40$ and I $\leq$ theoretical frequency $(T)<5$, the Fisher exact test was used.

Abbreviations: KPS, Karnofsky performance status; 2DRT, two-dimensional conformal radiation therapy; 3DRT, three-dimensional conformal radiation therapy; IMRT, intensity-modulated radiation therapy.

multivariate analysis to prevent multicollinearity. For instance, ypTNM was obviously correlated with ypT stage and ypN stage, and thus ypTNM was not included in the multivariate analysis. Finally, the percentage decrease in maximal esophageal wall thickness, ypT stage, ypN stage, PNI, and LVI were included in the multivariate analysis. The results showed that a $\leq 40 \%$ decrease of maximal esophageal wall thickness (HR: 1.907, 95\% CI: $1.149-3.165 ; \mathrm{p}=0.012$ ) and PNI (HR: 2.138, 95\% CI: $1.094-4.178 ; \mathrm{P}=0.026$ ) were independent adverse prognosticators for OS. The 5-year OS was significantly higher in patients with $>40 \%$ decrease of maximal esophageal wall thickness than in those with a $\leq 40 \%$ decrease $(60.1 \%$ vs $29.0 \% ; \mathrm{P}=0.005$; Figure $2 \mathrm{~A})$.

For DFS, the percentage decrease of maximal esophageal wall thickness, ypT stage, ypN stage, ypTNM, PNI, and LVI showed significant influence in the univariable analyses $(\mathrm{P}<0.05$ for all $)$ (Table 4). Multivariate analysis showed that the significant predictors of DFS were a $\leq 40 \%$ decrease of maximal esophageal wall thickness (HR: 2.054, 95\% CI: $1.288-3.277 ; \mathrm{P}=0.003$ ), LVI (HR: 2.874, 95\% CI: 1.574-5.248; $\mathrm{P}=0.001$ ), and PNI (HR: 2.050; 95\% CI: $1.044-4.023 ; \mathrm{P}=0.037$ ). Patients with $>40 \%$ 

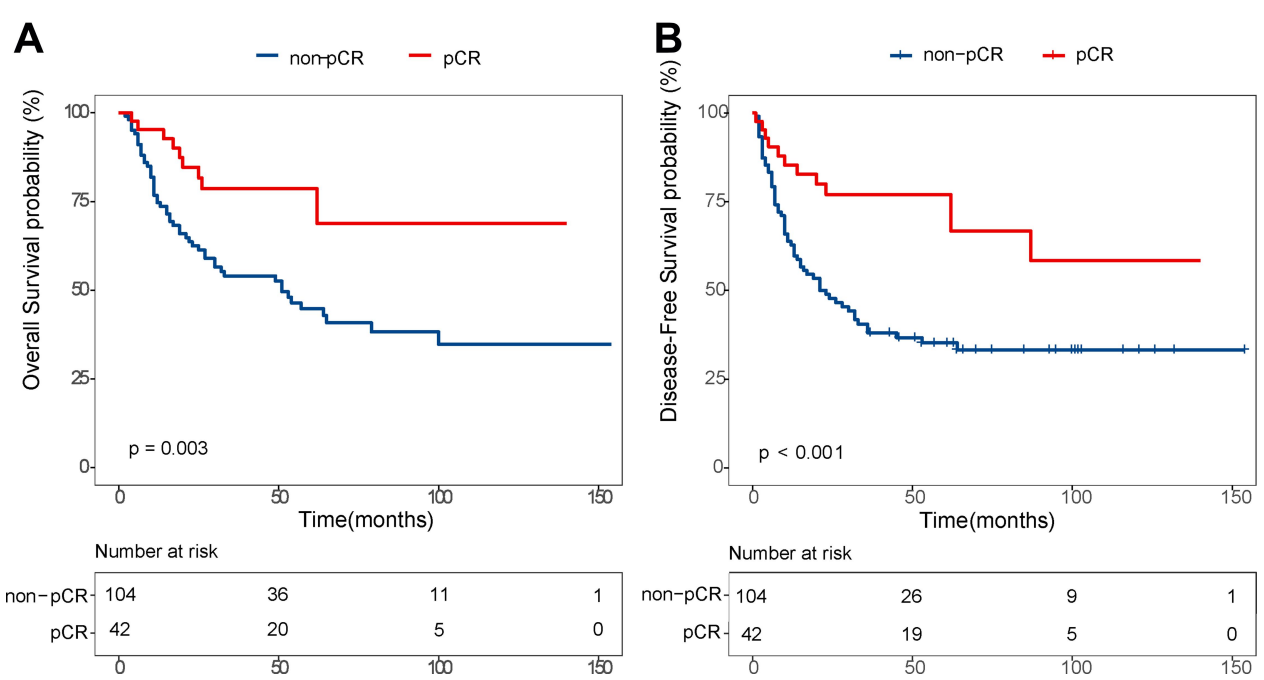

Figure I Comparison of overall (A), disease-free survival (B) between PCR group and non-pCR.

percentage decrease of maximal esophageal wall thickness had a better 5 -year DFS than those with a $\leq 40 \%$ decrease (54.4\% vs $27.8 \%$; Figure 2B).

\section{Recurrence Site}

Tumor recurrence occurred in 52/146 (35.6\%) patients; these included including 40 loco-regional recurrences and 35 distant recurrences (Table 5). Among those with locoregional recurrences, more than half $(35 / 40)$ presented with recurrence in the regional lymph nodes, including cervical (17/40), mediastinal (19/40), and abdominal lymph nodes (12/40). Meanwhile, the most frequent sites of distant metastases were lung (18/35), bone (15/35), and liver (10/35). The percentage decrease of maximal esophageal wall thickness was corrected with tumor recurrence. Patients with $>40 \%$ decrease of maximal esophageal wall thickness were 3.229 times more likely to have cervical lymph node recurrence than patients with $\leq 40 \%$ decrease $(\mathrm{P}=0.037)$.
Meanwhile, the rates of mediastinal and abdominal lymph node recurrences were similar between those with $>40 \%$ and with $\leq 40 \%$ decrease. Meanwhile, there was a significant difference in distal recurrence. Patients who developed liver and bone metastasis were more likely to have $<40 \%$ decrease of maximal esophageal wall thickness than $\geq 40 \%$ (HR: 5.22, $\mathrm{P}=0.0461$; HR: $3.683, \mathrm{P}=0.033$, respectively).

\section{Recurrence Time and Frequency}

The median time to the first recurrence was 9 months (range, 1-45 months) in the overall population, while it was 8 months (range, 1-45 months) and 10 months (range, 2-32 months) for those with a $<40 \%$ and $\geq 40 \%$ decrease of maximal esophageal wall thickness, respectively. Figure 3 shows the frequency of recurrence according to the percentage decrease of maximal esophageal wall thickness. All patients with $\geq 40 \%$ decrease of maximal esophageal wall thickness developed recurrence within 3 years of surgery. Meanwhile,

Table 2 Multivariable Logistic Analyses for PCR

\begin{tabular}{|l|l|l|l|}
\hline & HR & $\mathbf{9 5 \%}$ Cl & P \\
\hline Sex & & & \\
\hline Female vs male & 0.283 & $0.097-0.827$ & \\
\hline Decrease of maximal esophageal wall thickness (\%) & & & \\
\hline$\leq 40 \%$ vs $>40 \%$ & 2.022 & $1.005-4.930$ \\
\hline Radiation dose (Gy) & & & 0.049 \\
\hline$<40$ vs $\geq 40$ & 2.784 & $0.971-7.977$ \\
\hline
\end{tabular}

Abbreviations: $\mathrm{HR}$, hazard ratio; $\mathrm{Cl}$, confidence interval. 
Table 3 Predictors of Overall Survival in ESCC Patients After Neoadjuvant Chemoradiotherapy and Surgery

\begin{tabular}{|c|c|c|c|c|}
\hline \multirow[t]{2}{*}{ Clinicopathologic Parameters } & \multicolumn{4}{|c|}{ Univariate Analysis Multivariate Analysis } \\
\hline & HR (95\% Cl) & $\mathbf{P}$ & HR (95\% Cl) & $\mathbf{P}$ \\
\hline \multicolumn{5}{|l|}{ Age } \\
\hline$\leq 55$ vs $>55$ & I.644(0.659-4.102) & 0.287 & & \\
\hline \multicolumn{5}{|l|}{ Sex } \\
\hline Female vs Male & $0.867(0.527-1.427)$ & 0.575 & & \\
\hline \multicolumn{5}{|l|}{ Smoking history } \\
\hline No vs Yes & $1.193(0.723-1.969)$ & 0.489 & & \\
\hline \multicolumn{5}{|c|}{ Decrease of maximal esophageal wall thickness (\%) } \\
\hline$\leq 40 \%$ vs $>40 \%$ & $2.017(1.223-3.326)$ & 0.006 & $1.907(1.149-3.165)$ & 0.012 \\
\hline \multicolumn{5}{|l|}{ Tumor location } \\
\hline Upper vs Middle/Distal & $1.569(0.877-2.807)$ & 0.129 & & \\
\hline \multicolumn{5}{|l|}{ Clinical T stage } \\
\hline $\mathrm{T} 2$ vs T3/T4 & $0.65 I(0.089-4.74)$ & 0.672 & & \\
\hline \multicolumn{5}{|l|}{ Clinical $\mathrm{N}$ stage } \\
\hline No vs $\mathrm{N}+$ & $2.008(0.914-4413)$ & 0.083 & & \\
\hline \multicolumn{5}{|l|}{ Clinical TNM stage } \\
\hline II vs III/IV & $1.770(0.710-4.415)$ & 0.221 & & \\
\hline \multicolumn{5}{|l|}{ yp T stage } \\
\hline T0-2 vs T3-4 & $2.133(1.298-3.505)$ & 0.003 & $1.36 \mathrm{I}(0.780-2.373)$ & 0.278 \\
\hline \multicolumn{5}{|l|}{ yp $N$ stage } \\
\hline No vs N+ & $1.959(1.194-3.216)$ & 0.008 & I.487(0.869-2.542) & 0.148 \\
\hline \multicolumn{5}{|l|}{ ур TNM stage } \\
\hline I/II vs III/VI & $2.599(1.566-4.312)$ & $<0.001$ & & \\
\hline \multicolumn{5}{|l|}{ Radiation dose (Gy) } \\
\hline$<40$ vs $\geq 40$ & $0.713(0.412-1.232)$ & 0.225 & & \\
\hline \multicolumn{5}{|l|}{ Chemotherapy cycle } \\
\hline I vs $\geq 2$ & $0.682(0.4 I-I .132)$ & 0.138 & & \\
\hline \multicolumn{5}{|l|}{ Number of $\mathrm{LN}$ examined } \\
\hline$<25$ vs $\geq 25$ & I.I58(0.705-I.902) & 0.563 & & \\
\hline \multicolumn{5}{|l|}{ Lymph vessel invasion } \\
\hline No vs Yes & $2.498(I .4 I I-4.420)$ & 0.002 & $1.729(0.921-3.247)$ & 0.088 \\
\hline \multicolumn{5}{|l|}{ Perineural invasion } \\
\hline No vs Yes & $2.977(1.597-5.548)$ & 0.001 & $2.138(1.094-4.178)$ & 0.026 \\
\hline
\end{tabular}


Table 3 (Continued).

\begin{tabular}{|l|l|l|l|l|}
\hline \multirow{2}{*}{ Clinicopathologic Parameters } & \multicolumn{3}{l|}{ Univariate Analysis Multivariate Analysis } \\
\cline { 2 - 5 } & HR (95\% Cl) & P & HR (95\% Cl) \\
\hline Adjuvant chemotherapy & & & & P \\
\hline No vs Yes & $1.626(0.99 I-2.669)$ & 0.054 & \\
\hline
\end{tabular}

Abbreviations: $\mathrm{HR}$, hazard ratio; $\mathrm{Cl}$, confidence interval.

although most patients (84.6\%) with a $<40 \%$ decrease also developed recurrence within 3 years of surgery, 6 patients (15.4\%) developed recurrence more than 3 years after surgery. In addition, among patients with $\geq 60 \%$ decrease of maximal esophageal wall thickness, only 5 patients developed recurrence, and all recurrences occurred within the 2 years after surgery.

\section{Discussion}

The usefulness of the change in maximal esophageal wall thickness for predicting pCR, survival, and recurrence in locally advanced ESCC patients undergoing NCRT followed by surgery remains unclear to date. In this study, a $\leq 40 \%$ decrease of maximal esophageal wall thickness after NCRT was strongly associated with a low pCR rate, short survival time, and a high risk of recurrence.

A
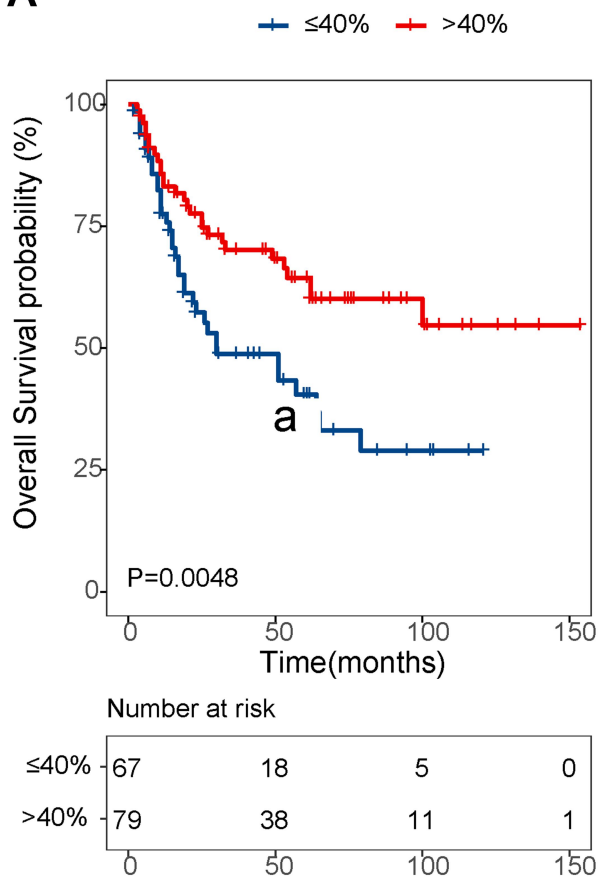

Surgery combined with NCRT has become the standard treatment modality for locally advanced EC. Nearly onethird of patients who undergo NCRT achieve pCR. ${ }^{9,13}$ The treatment response to NCRT is crucial for selecting subsequent treatment and preventing overtreatment, and thus its accurate evaluation is crucial. Several studies have attempted to establish effective methods to predict $\mathrm{pCR} .{ }^{14} \mathrm{~A}$ recent multicenter prospective study found that changes in $18 \mathrm{~F}$ FDG positron emission tomography (PET)-CT after NCRT could identify pCR in esophageal cancer. ${ }^{15}$ However, PET is not commonly used in clinical practice because of its high cost; CT is more commonly performed.

Swisher et al and Li et al showed that pre- or postchemoradiotherapy maximal esophageal wall thickness was associated with the response to chemoradiotherapy and survival. ${ }^{11,12}$ Furthermore, Djuric-Stefanovic et al found that

\section{B}
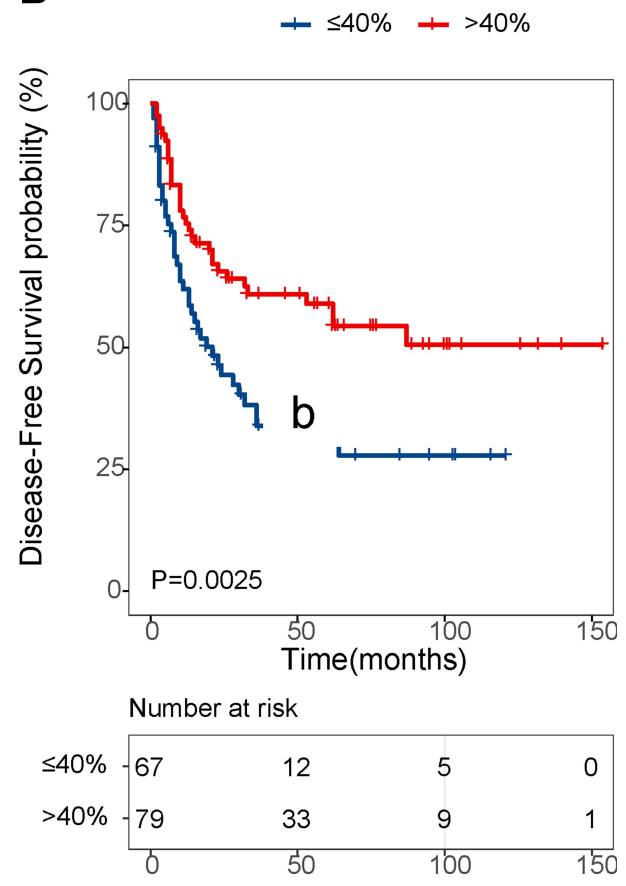

Figure 2 Comparison of overall (A), disease-free survival (B) according to the percentage decrease of maximal esophageal wall thickness. 
Table 4 Predictors of Disease-Free Survival in ESCC Patients After Neoadjuvant Chemoradiotherapy and Surgery

\begin{tabular}{|c|c|c|c|c|}
\hline \multirow[t]{2}{*}{ Clinicopathologic Parameters } & \multicolumn{4}{|c|}{ Univariate Analysis Multivariate Analysis } \\
\hline & HR (95\% Cl) & $\mathbf{p}$ & HR (95\% Cl) & $\mathbf{p}$ \\
\hline \multicolumn{5}{|l|}{ Age } \\
\hline$\leq 55$ vs $>55$ & $0.930(0.586-1.477)$ & 0.759 & & \\
\hline \multicolumn{5}{|l|}{ Sex } \\
\hline Female vs Male & I.777(0.77I-4.098) & 0.177 & & \\
\hline \multicolumn{5}{|l|}{ Smoking history } \\
\hline No vs Yes & $1.06 I(0.67 I-1.678)$ & 0.799 & & \\
\hline \multicolumn{5}{|c|}{ Decrease of maximal esophageal wall thickness (\%) } \\
\hline$\leq 40 \%$ vs $>40 \%$ & $1.994(1.258-3.161)$ & 0.003 & $2.054(1.288-3.277)$ & 0.003 \\
\hline \multicolumn{5}{|l|}{ Tumor location } \\
\hline Upper vs Middle/Distal & $1.339(0.800-2.242)$ & 0.266 & & \\
\hline \multicolumn{5}{|l|}{ Clinical T stage } \\
\hline $\mathrm{T} 2$ vs $\mathrm{T} 3 / \mathrm{T} 4$ & $0.860(0.119-6.230)$ & 0.881 & & \\
\hline \multicolumn{5}{|l|}{ Clinical $\mathrm{N}$ stage } \\
\hline $\mathrm{N} 0$ vs $\mathrm{N}+$ & $1.966(0.978-3.951)$ & 0.058 & & \\
\hline \multicolumn{5}{|l|}{ Clinical TNM stage } \\
\hline II vs III/IV & $1.908(0.828-4.399)$ & 0.129 & & \\
\hline \multicolumn{5}{|l|}{ ур T stage } \\
\hline T0-2 vs T3-4 & $2.088(1.32 I-3.302)$ & 0.002 & I.167(0.658-2.070) & 0.598 \\
\hline \multicolumn{5}{|l|}{ yр T stage } \\
\hline No vs $\mathrm{N}+$ & $1.775(1.123-2.806)$ & 0.014 & $1.263(0.759-2.103)$ & 0.368 \\
\hline \multicolumn{5}{|l|}{ yp TNM stage } \\
\hline I/II vs III/VI & $2.208(1.391-3.505)$ & 0.001 & & \\
\hline \multicolumn{5}{|l|}{ Radiation dose(Gy) } \\
\hline$<40$ vs $\geq 40$ & $0.656(0.398-1.080)$ & 0.098 & & \\
\hline \multicolumn{5}{|l|}{ Chemotherapy cycle } \\
\hline I vs $\geq 2$ & $0.77 \mid(0.47-\mid .240)$ & 0.283 & & \\
\hline \multicolumn{5}{|l|}{ Number of LN examined } \\
\hline$<25$ vs $\geq 25$ & $1.028(0.649-1.626)$ & 0.908 & & \\
\hline \multicolumn{5}{|l|}{ Lymph vessel invasion } \\
\hline No vs Yes & $3.442(2.037-5.817)$ & $<0.001$ & $2.874(1.574-5.248)$ & 0.001 \\
\hline
\end{tabular}


Table 4 (Continued).

\begin{tabular}{|l|l|l|l|l|}
\hline \multirow{2}{*}{ Clinicopathologic Parameters } & \multicolumn{3}{l|}{ Univariate Analysis Multivariate Analysis } \\
\cline { 2 - 5 } & HR (95\% Cl) & $\mathbf{P}$ & HR (95\% CI) \\
\hline Perineural invasion & & & & \\
\hline No vs Yes & $2.543(1.413-4.575)$ & 0.002 & $2.050(1.044-4.023)$ \\
\hline Adjuvant chemotherapy & & & & 0.037 \\
\hline No vs Yes & $1.491(0.945-2.354)$ & 0.086 & & \\
\hline
\end{tabular}

Abbreviations: $\mathrm{HR}$, hazard ratio; $\mathrm{Cl}$, confidence interval.

Table 5 Tumor Recurrence Site According to the Percent of Decrease of Maximal Esophageal Wall Thickness

\begin{tabular}{|c|c|c|c|c|c|}
\hline Site of Recurrence & Total $(n=\mid 46, \%)$ & $\leq 40 \%(n=67, \%)$ & $>40 \%(n=79, \%)$ & HR (95\% Cl) & $\mathbf{P}$ \\
\hline Locoregional recurrence & $40(27.4)$ & $25(37.3)$ & $15(19.0)$ & $2.540(1.20 \mathrm{I}-5.372)$ & 0.015 \\
\hline Anastomosis & $7(4.8)$ & $5(7.5)$ & $2(2.5)$ & $3.105(0.582-16.553)$ & 0.185 \\
\hline Regional lymph node & $35(24.0)$ & $21(31.3)$ & $14(17.7)$ & $2.120(0.977-4.599)$ & 0.057 \\
\hline Cervical & $17(11.6)$ & $12(17.9)$ & $5(6.3)$ & 3.229 (1.075-9.107) & 0.037 \\
\hline Mediastinum & $19(13.0)$ & $12(17.9)$ & $7(8.9)$ & $2.244(0.829-6.076)$ & 0.112 \\
\hline Abdominal & $12(8.2)$ & $7(10.4)$ & $5(6.3)$ & $1.727(0.522-5.716)$ & 0.371 \\
\hline Distant recurrence & $35(24.0)$ & $19(28.4)$ & $13(16.5)$ & $2.010(0.905-4.46 \mathrm{I})$ & 0.086 \\
\hline Liver & $10(6.8)$ & $8(11.9)$ & $2(2.5)$ & $5.220(1.069-25.501)$ & 0.041 \\
\hline Lung & $18(12.3)$ & II (I6.4) & $7(8.9)$ & $2.020(0.736-5.547)$ & 0.172 \\
\hline Bone & $15(10.3)$ & II (I6.4) & $4(5.1)$ & $3.683(1.114-12.174)$ & 0.033 \\
\hline Others & $5(3.4)$ & $2(3.0)$ & $3(2.3)$ & $0.779(0.126-4.809)$ & 0.788 \\
\hline
\end{tabular}

a significant correlation between post-NCRT maximal esophageal wall thickness measured via CT and pCR. Further, they reported that the combination of maximal esophageal wall thickness $\leq 9 \mathrm{~mm}$ and average post-contrast density $\leq 64 \mathrm{HU}$ could correctly predict pCR. ${ }^{16}$ However, the results of their studies did not show whether patients with a higher percentage decrease of maximal esophageal wall thickness had a better response to NCRT and survival. ${ }^{11,12,16}$ Evaluation of the therapeutic response and prognosis solely by pre- or postchemoradiotherapy maximal esophageal wall thickness is often influenced by individualized differences. In contrast, the percentage decrease of maximal esophageal wall thickness is less influenced by patient characteristics, and thus it may have more prognostic value.

Many studies have reported that $\mathrm{pCR}$ is a favorable factor for improving survival and reducing recurrence. ${ }^{17,18}$ Similarly, the pCR group in our study demonstrated significantly better 5-year OS and DFS than did the non-pCR group. Although there had been several studies on the relevant factors affecting $\mathrm{pCR},{ }^{19-21}$ few had investigated the predictive value of the percentage decrease of maximal esophageal wall thickness for ESCC treated with NCRT. In this study, the pCR rate was significantly higher in those with a $>40 \%$ decrease of maximal esophageal wall thickness than in those with $\leq 40 \%$. A previous study by $\mathrm{Li}$ et $\mathrm{al}^{12}$ showed that treatment response to chemoradiotherapy was significantly correlated with pretreatment maximal esophageal wall thickness among patients with locally advanced ESCC. However, there was no significant difference in treatment response according to the percentage decrease of esophageal wall thickness. One possible explanation for this result was that their study divided the percentage decrease of esophageal wall thickness into $\geq 10 \%$ 


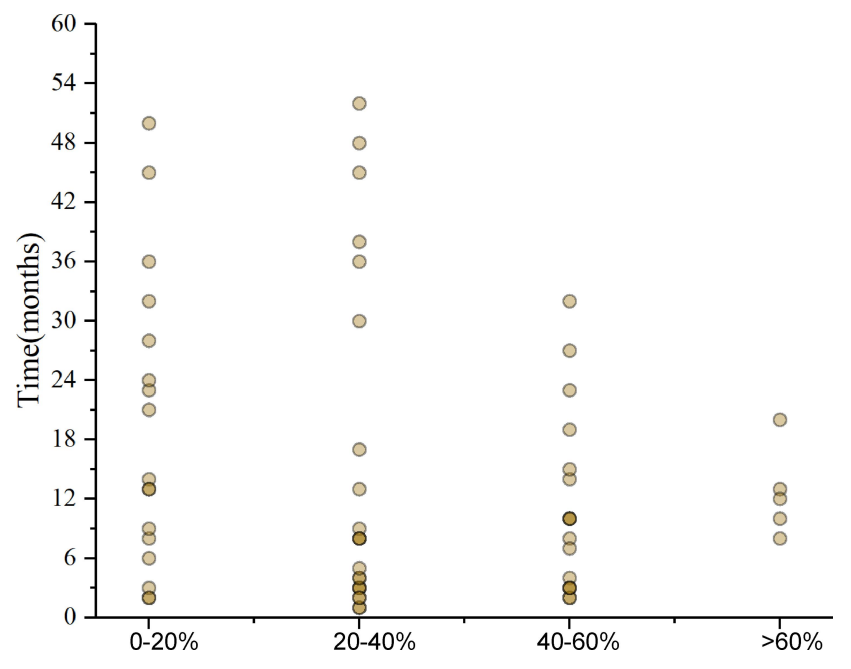

Figure 3 Frequency of recurrences in different percentage decrease of maximal esophageal wall thickness.

and $<10 \%$. In addition, their study had a small sample size of only 91 cases, which may have affected the results.

Our result may provide a new perspective that patients with a $>40 \%$ decrease of esophageal wall thickness could be safely followed through active surveillance without surgery. However, 51 of the 71 patients with $>40 \%$ decrease of esophageal wall thickness did not achieve pCR after NCRT, and 14 of the 67 patients with $\leq 40 \%$ decrease achieved pCR. This indicates that the percentage decrease of esophageal wall thickness alone is inadequate to predict pCR. In addition, most studies showed that endoscopic biopsy, CT, PET, and endoscopic ultrasonography lack precision for evaluating tumor response to NCRT. ${ }^{14,22-24}$ Given the lack of effective methods to accurately predict $\mathrm{pCR}$, international guidelines recommend esophageal resection in all patients after neoadjuvant therapy. ${ }^{25}$ Our results provide new perspectives and warrant further research.

In addition to treatment response, we also found that the percentage decrease of esophageal wall thickness was correlated with tumor recurrence. Patients with a $>40 \%$ decrease of maximal esophageal wall thickness were 3.229 times more likely to have cervical lymph node recurrence than patients with a $\leq 40 \%$ decrease. Furthermore, there was a significant difference in the rate of distal recurrence according to the percentage decrease. Patients who developed liver and bone metastasis were more likely to have $<40 \%$ decrease of maximal esophageal wall thickness than $\geq 40 \%$. With respect to recurrence time and frequency, as expected, recurrences were more frequent in those with a $\leq 40 \%$ decrease of maximal esophageal wall thickness than those with a $>40 \%$ decrease. Thus, more intensive follow-up of the percentage decrease of maximal esophageal wall thickness $\leq 40 \%$ may be needed. Furthermore, although most of the patients developed recurrence within 3 years after surgery, some of the patients with $\leq 40 \%$ decrease of maximal esophageal wall thickness developed recurrence more than 3 years after surgery. Therefore, we recommend active surveillance for the first 3 years after surgery, which complies with the current National Comprehensive Cancer Network guidelines. For patients with $\leq 40 \%$ decrease of maximal esophageal wall thickness, we recommend prolonging the postoperative surveillance time to more than 5 years.

PNI was also identified to be an independent prognostic factor of both OS and DFS in our study. Lagarde et al and $\mathrm{Tu}$ et al concluded that the presence of PNI had an adverse impact on survival time. ${ }^{26,27}$ In addition, we found that LVI was independently associated with a shorter DFS. Previous studies by Lagarde et al and $\mathrm{Gu}$ et al proposed that LVI was an indicator of adverse prognosis. $^{27,28}$ Collectively, these findings support the predictive value of PNI and LVI for OS and DFS in ESCC.

This study had some limitations. It was a single-center retrospective study with a small sample size. Prospective studies in a larger number of patients are needed to confirm our results. Furthermore, other important covariables such as baseline body weight status or PET data were lacking. Thus, we could not evaluate the incremental benefit from the change in maximal thickness. In addition, we exclusively evaluated ESCC patients, and thus the generalizability of our findings to patients with other adenocarcinoma warrants additional investigation.

\section{Conclusion}

The percentage decrease of maximal esophageal wall thickness is independently associated with pCR and recurrence among ESCC patients who undergo NCRT and surgery, thus making it a potential predictive factor. Treatment decisions following NCRT and postoperative surveillance strategies may be individualized according to the preoperative percentage decrease of maximal esophageal wall thickness. 


\section{Abbreviations}

ESCC, esophageal squamous cell carcinoma; CT, computed tomography; 2DRT, two-dimensional conformal radiation therapy; HR, hazard ratio; CI, confidence interval.

\section{Data Sharing Statement}

The data used to support the findings of this study are included with the article and the supplementary file (Additional file 1).

\section{Ethics Approval and Consent to Participate}

This retrospective study was approved by the ethics committee of the Fujian Province Cancer Hospital (YKT2020017-01) and conducted in accordance with the principles of the Declaration of Helsinki and its amendment. All patients provided written informed consent prior to treatment, and all information was anonymized prior to analysis.

\section{Acknowledgments}

The authors thank all patients who participated in the present study.

\section{Author Contributions}

All authors made a significant contribution to the work reported, whether that is in the conception, study design, execution, acquisition of data, analysis and interpretation, or in all these areas; took part in drafting, revising or critically reviewing the article; gave final approval of the version to be published; have agreed on the journal to which the article has been submitted; and agree to be accountable for all aspects of the work.

\section{Funding}

This study was supported in part by grants from Innovation of Science and Technology, Fujian Province (Grant number: 2018Y9111) and the Financial Foundation of Fujian Province (Grant number: (2019)827).

\section{Disclosure}

The authors declare that the submitted work was not carried out in the presence of any personal, professional, or financial relationships that could potentially be construed as a conflict of interest.

\section{References}

1. Bray F, Ferlay J, Soerjomataram I, et al. Global cancer statistics 2018: GLOBOCAN estimates of incidence and mortality worldwide for 36 cancers in 185 countries. CA Cancer J Clin. 2018;68 (6):394-424. doi:10.3322/caac.21492

2. Kamangar F, Nasrollahzadeh D, Safiri S, et al. The global, regional, and national burden of oesophageal cancer and its attributable risk factors in 195 countries and territories, 1990-2017: a systematic analysis for the Global Burden of Disease Study 2017. Lancet Gastroenterol Hepatol. 2020;5(6):582-597. doi:10.1016/s24681253(20)30007-8

3. Leng X-F, Daiko H, Han Y-T, Mao Y-S. Optimal preoperative neoadjuvant therapy for resectable locally advanced esophageal squamous cell carcinoma. Ann N Y Acad Sci. 2020;1482(1):213-224. doi:10.1111/nyas. 14508

4. Ronellenfitsch U, Schwarzbach M, Hofheinz R, et al. Perioperative chemo(radio)therapy versus primary surgery for resectable adenocarcinoma of the stomach, gastroesophageal junction, and lower esophagus. Cochrane Database Syst Rev. 2013;CD008107(5). doi:10.1002/14651858.CD008107.pub2

5. Donohoe CL, McGillycuddy E, Reynolds JV. Long-term health-related quality of life for disease-free esophageal cancer patients. World J Surg. 2011;35(8):1853-1860. doi:10.1007/s00268011-1123-6

6. Herskovic A, Russell W, Liptay M, Fidler MJ, Al-Sarraf M. Esophageal carcinoma advances in treatment results for locally advanced disease: review. Ann Oncol. 2012;23(5):1095-1103. doi:10.1093/annonc/mdr433

7. Steffen T, Dietrich D, Schnider A, et al. Recurrence patterns and long-term results after induction chemotherapy, chemoradiotherapy, and curative surgery in patients with locally advanced Esophageal cancer. Ann Surg. 2019;269(1):83-87. doi:10.1097/sla.0000 000000002435

8. Yamashita K, Hosoda K, Moriya H, et al. Prognostic advantage of docetaxel/cisplatin/5-fluorouracil neoadjuvant chemotherapy in clinical stage II/III Esophageal squamous cell carcinoma due to excellent control of preoperative disease and postoperative lymph node recurrence. Oncology. 2017;92(4):221-228. doi:10.1159/000455128

9. Shapiro J, van Lanschot JJB, Hulshof MCCM, et al. Neoadjuvant chemoradiotherapy plus surgery versus surgery alone for oesophageal or junctional cancer (CROSS): long-term results of a randomised controlled trial. Lancet Oncol. 2015;16(9):1090-1098. doi:10.1016/ S1470-2045(15)00040-6

10. Noordman BJ, Spaander MCW, Valkema R, et al. Detection of residual disease after neoadjuvant chemoradiotherapy for oesophageal cancer (preSANO): a prospective multicentre, diagnostic cohort study. Lancet Oncol. 2018;19(7):965-974. doi:10.1016/S14702045(18)30201-8

11. Swisher SG, Maish M, Erasmus JJ, et al. Utility of PET, CT, and EUS to identify pathologic responders in esophageal cancer. Ann Thorac Surg. 2004;78(4):1152-1160. doi:10.1016/j.athoracsur.2004.04.046

12. Li S-H, Rau K-M, Lu H-I, et al. Pre-treatment maximal oesophageal wall thickness is independently associated with response to chemoradiotherapy in patients with T3-4 oesophageal squamous cell carcinoma. Eur J Cardiothorac Surg. 2012;42(6):958-964. doi:10.1093/ ejcts/ezs 136

13. van Hagen P, Hulshof MCCM, van Lanschot JJB, et al. Preoperative chemoradiotherapy for esophageal or junctional cancer. $N$ Engl $J$ Med. 2012;366(22):2074-2084. doi:10.1056/NEJMoa1112088

14. de Gouw D, Klarenbeek BR, Driessen M, et al. Detecting pathological complete response in Esophageal cancer after neoadjuvant therapy based on imaging techniques: a diagnostic systematic review and meta-analysis. J Thorac Oncol. 2019;14(7):1156-1171. doi:10.1016/ j.jtho.2019.04.004 
15. Borggreve AS, Goense L, van Rossum PSN, et al. Preoperative prediction of pathologic response to neoadjuvant chemoradiotherapy in patients with esophageal cancer using 18F-FDG PET/CT and DWMRI: a Prospective Multicenter Study. Int J Radiat Oncol Biol Phys. 2020;106(5):998-1009. doi:10.1016/j.ijrobp.2019.12.038

16. Djuric-Stefanovic A, Jankovic A, Saponjski D, et al. Analyzing the post-contrast attenuation of the esophageal wall on routine contrast-enhanced MDCT examination can improve the diagnostic accuracy in response evaluation of the squamous cell esophageal carcinoma to neoadjuvant chemoradiotherapy in comparison with the esophageal wall thickness. Abdom Radiol. 2019;44 (5):1722-1733. doi:10.1007/s00261-019-01911-w

17. Barbetta A, Sihag S, Nobel T, et al. Patterns and risk of recurrence in patients with esophageal cancer with a pathologic complete response after chemoradiotherapy followed by surgery. J Thorac Cardiovasc Surg. 2019;157(3):1249-1259 e1245. doi:10.1016/j.jtcvs.20 18.09.136

18. Heneghan HM, Donohoe C, Elliot J, et al. Can CT-PET and endoscopic assessment post-neoadjuvant chemoradiotherapy predict residual disease in Esophageal cancer? Ann Surg. 2016;264(5):831-838. doi:10.1097/SLA.0000000000001902

19. Tomasello G, Petrelli F, Ghidini M, et al. Tumor regression grade and survival after neoadjuvant treatment in gastro-esophageal cancer: a meta-analysis of 17 published studies. Eur J Surg Oncol. 2017;43 (9):1607-1616. doi:10.1016/j.ejso.2017.03.001

20. Thomas M, Borggreve AS, van Rossum PSN, et al. Radiation dose and pathological response in oesophageal cancer patients treated with neoadjuvant chemoradiotherapy followed by surgery: a multi-institutional analysis. Acta Oncol. 2019;58(10):1358-1365. doi:10.1080/0284186X.2019.1646432

21. Haisley KR, Laird AE, Nabavizadeh N, et al. Association of intervals between neoadjuvant chemoradiation and surgical resection with pathologic complete response and survival in patients with Esophageal cancer. JAMA Surgery. 2016;151(11):e162743. doi:10.1001/jamasurg.2016.2743
22. van der Bogt R, Noordman BJ, Krishnadath KK, et al. Endoscopic ultrasound measurements for detection of residual disease after neoadjuvant chemoradiotherapy for esophageal cancer. Endoscopy. 2019;51(4):326-332. doi:10.1055/a-0795-3220

23. van Rossum PSN, Goense L, Meziani J, et al. Endoscopic biopsy and EUS for the detection of pathologic complete response after neoadjuvant chemoradiotherapy in esophageal cancer: a systematic review and meta-analysis. Gastrointest Endosc. 2016;83(5):866-879. doi:10.1016/j.gie.2015.11.026

24. Wang L, Liu L, Han C, et al. The diffusion-weighted magnetic resonance imaging (DWI) predicts the early response of esophageal squamous cell carcinoma to concurrent chemoradiotherapy. Radiother Oncol. 2016;121(2):246-251. doi:10.1016/j.radonc.2016.10.021

25. Little AG, Lerut AE, Harpole DH, et al. The society of thoracic surgeons practice guidelines on the role of multimodality treatment for cancer of the esophagus and gastroesophageal junction. Ann Thorac Surg. 2014;98(5):1880-1885. doi:10.1016/j.athoracsur.2014.07.069

26. Tu -C-C, Hsu P-K, Chien L-I, et al. Prognostic histological factors in patients with esophageal squamous cell carcinoma after preoperative chemoradiation followed by surgery. BMC Cancer. 2017;17(1):62. doi:10.1186/s12885-017-3063-5

27. Lagarde SM, Phillips AW, Navidi M, et al. The presence of lymphovascular and perineural infiltration after neoadjuvant therapy and oesophagectomy identifies patients at high risk for recurrence. $\mathrm{Br}$ $J$ Cancer. 2015;113(10):1427-1433. doi:10.1038/bjc.2015.354

28. Gu Y-M, Yang Y-S, Hu W-P, et al. Prognostic value of lymphovascular invasion in patients with esophageal squamous cell carcinoma. Ann Transl Med. 2019;7(12):256. doi:10.21037/atm.2019.05.23

\section{Publish your work in this journal}

Cancer Management and Research is an international, peer-reviewed open access journal focusing on cancer research and the optimal use of preventative and integrated treatment interventions to achieve improved outcomes, enhanced survival and quality of life for the cancer patient.
The manuscript management system is completely online and includes a very quick and fair peer-review system, which is all easy to use. Visit http://www.dovepress.com/testimonials.php to read real quotes from published authors. 